'Sección de Hematología. Hospital del Salvador. Santiago, Chile.

2Laboratorio Reumatología/

Inmunología. Hospital del Salvador. Santiago, Chile ${ }^{3}$ Servicio de Medicina. Hospital del Salvador. Santiago, Chile. ${ }^{4}$ The Binding Site. Buenos Aires, Argentina. aBioquímico. bTecnólogo médico 'PhD en Bioquímica, Inmunología y Virología.

Fuente de apoyo financiero: La compañía The Binding Site proporcionó los reactivos para la medición de cadenas livianas libres en suero, sin costo para el hospital.

Recibido el 6 de julio de 2017 , aceptado 19 de enero de 2018.

Correspondencia a: Camila Peña

Avenida Salvador 364, Providencia, Santiago, Chile. Teléfono: (56) - 25754000 - Fax (56) 225753795 camipena@gmail.com

\section{Sensibilidad diagnóstica de electroforesis de proteínas y cadenas livianas libres séricas en gammapatías monoclonales}

\author{
CAMILA PEÑA ${ }^{1}$, MANUELA ORTIZ, 2, JAVIER VOISIN ${ }^{3}$, \\ ALEXIS PERALTA ${ }^{2, a}$, VIVIANA BALBOA ${ }^{2, b}$, FLORENCIA DELGADO $^{4, \mathrm{a}, \mathrm{c}}$
} measurements for monoclonal gammopathies

Background: International guidelines suggest a screening panel for monoclonal gammopathies that contains serum protein electrophoresis (SPE), free light chain (FLC) measurements and immunofixation. This combination provides the possibility of a timely accurate diagnosis. Aim: To evaluate the sensibility of a simple screening panel (SPE + FLC). Material and Methods: We analyzed 191 consecutive serum samples of patients with a suspected monoclonal gammopathy (MG). Results: Seventy five patients were diagnosed with MG. The sensitivity and specificity of the combination of SPE + FLC for the diagnosis of monoclonal gammopathy were $95 \%$ (95\% confidence intervals 89-99) and 99\% (95\% confidence intervals 96-100), respectively. Conclusions: We were able to validate the international recommendations on the diagnostic accuracy of this simple combination of two tests in serum for monoclonal gammopathy.

(Rev Med Chile 2018; 146: 64-67)

Key words: Diagnosis; Multiple Myeloma; Paraproteinemias.

L as cadenas livianas libres (CLL) séricas son un importante marcador diagnóstico para las gammapatías monoclonales (GM) y, por más de 150 años, la presencia de proteínas de Bence Jones en orina fue indicador de producción monoclonal. Sin embargo, durante los últimos 15 años, ha habido un cambio de paradigma, gracias a la disponibilidad del inmunoensayo que mide kappa $(\kappa)$ y lambda $(\lambda)$ libres en suero en forma automatizada, específica y sensible ${ }^{1,2}$. De hecho, desde 2009, el International Myeloma Working Group (IMWG) recomienda la combinación de electroforesis de proteínas en suero (EFP), inmunofijación en suero (IFxs) y CLL en suero (CLLs) como tamizaje para diagnóstico de mieloma múltiple (MM) y otras GM, con la excepción de amiloidosis AL, donde aún es necesario la IFx en orina $^{3}$.

En nuestra realidad, la sospecha de GM suele ser investigada por un médico no hematólogo, quien solicita, en general, solo EFP, sin IFxs ni evaluaciones en orina. Surge así la necesidad de mejorar el panel diagnóstico. En este sentido, la comunidad internacional ha avalado el uso de EFP y CLLs ${ }^{3,4}$.

Nuestra institución forma parte de la red del sistema público chileno y no contábamos con la prueba de CLL al momento de realizar este estudio. 


\section{Objetivo}

Evaluar la sensibilidad, especificidad y likehood ratio (LR) del panel "EFP + CLLs" como prueba diagnóstica en pacientes con sospecha de GM comparado con el panel original solicitado $y$, cuando fuera factible, con el panel internacionalmente recomendado.

\section{Material y Método}

Se estudiaron prospectivamente 191 muestras consecutivas de pacientes con sospecha de GM, desde octubre de 2013 a abril de 2016. Se consideró gold standard diagnóstico el mielograma o biopsia con inmunohistoquímica compatible. Se definió panel solicitado (PS) como los exámenes solicitados por el médico tratante; $y$ panel internacional (PI) a la solicitud de lo recomendado por la IMWG (es decir EFP, IFx Y CLLs). A todos los PS se adicionó CLLs por nuestro equipo. Se midió sensibilidad del PS y PI, en los casos en que se contaba con este. Además, se analizó sensibilidad, especificidad y LR del panel a evaluar "EFP + CLLs". Las CLLs se cuantificaron según inserto (Freelite, The Binding Site Group Ltd, Birmingham, UK). El estudio fue autorizado por el Comité de Ética Científica del Hospital del Salvador.

\section{Resultados}

En el total de pacientes evaluados con sospecha de GM, la EFP fue solicitada en 191 casos (100\%), IFxs en $72(38 \%)$ e IFx en orina, solo en 38 pacientes (20\%). Las CLLs no estaban disponibles en el sistema público, por lo que el PI no se solicitó formalmente a ningún paciente.

De estos 191 pacientes, 75 tuvieron diagnóstico final de GM: 53 mieloma múltiple (39 MM cadena intacta, $12 \mathrm{MM}$ cadena liviana y $2 \mathrm{MM}$ no secretor), 8 plasmocitomas, 9 amiloidosis $\mathrm{AL}$ y 5 macroglobulinemia de Waldenström (MW).

La sensibilidad del panel "EFP + CLLs" en los 75 pacientes fue de $94,5 \%$ (95\% CI 89 a 99), la especificidad $98,6 \%$ (95\% CI 96 a 100$)$ y el LR (+) 110 (CI 95\% 15,72 a 780,32) y LR (-) 0,05 (CI $95 \% 0,02$ a 0,13). La sensibilidad del PS fue de $80 \%$ (95\% CI 69 a 88). Solo en 48 de los 75 pacientes con GM (64\%) se pudo analizar el PI con una sensibilidad para GM de 95,8\% (CI 95\% 85,7 a 99,4) (Tabla 1).

\section{Discusión}

El panel recomendado por la IMWG no es ampliamente utilizado en nuestra institución. La idea de simplificar el panel de tamizaje no es nueva y hay varios estudios que lo postulan. Antes de 2009,

Tabla 1

\begin{tabular}{|c|c|c|c|c|c|}
\hline & General & MM & Plasmocitoma & Amiloidosis & Waldenstöm \\
\hline $\operatorname{FFP}(+)\left(n^{\circ}\right)$ & 55 de 75 & 43 de 53 & 5 de 8 & 2 de 9 & 5 de 5 \\
\hline EFP Sensib. (\%) & $73,3(61,8$ a 82,8$)$ & $81,1(68,0390,5)$ & $62,5(24,4$ a 91,4$)$ & $22,2(2,8360)$ & $100(47,8$ a 100$)$ \\
\hline PS $(+)$ & 60 de 75 & 48 de 53 & 5 de 8 & 2 de 9 & 5 de 5 \\
\hline PS Sensib. (\%) & $80(69,1$ a 88,3$)$ & $90,5(79,3$ a 96,8$)$ & $62,5(24,4$ a 91,4$)$ & $22,2(2,8$ a 60$)$ & $100(47,8$ a 100$)$ \\
\hline $\mathrm{PS}+\mathrm{sFLC}(+)$ & 70 de 75 & 52 de 53 & 7 de 8 & 6 de 9 & 5 de 5 \\
\hline $\begin{array}{l}\text { PS+sFLC Sensib. } \\
(\%)\end{array}$ & $93,3(85,1$ a 97,8$)$ & $98,1(89,9$ a 99,9$)$ & $87,5(47,3$ a 99,6$)$ & $66,6(29,9$ a 92,5$)$ & $100(47,8$ a 100$)$ \\
\hline $\mathrm{EFP}+\mathrm{sFLC}(+)$ & 71 de 75 & 52 de 53 & 7 de 8 & 7 de 9 & 5 de 5 \\
\hline $\begin{array}{l}\text { EFP + sFLC Sen- } \\
\text { sib. (\%) }\end{array}$ & $94,6(86,9$ a 98,5$)$ & $98,1(89,9$ a 99,9$)$ & $87,5(47,3$ a 99,6$)$ & $77,7(39,9$ a 97,1$)$ & $100(47,8$ a 100$)$ \\
\hline $\mathrm{PI}(+)$ & 46 de 48 & 35 de 36 & 2 de 2 & 3 de 5 & 5 de 5 \\
\hline PI Sensib. (\%) & $95,8(85,7$ a 99,4$)$ & $100(93,2$ a 100$)$ & $100(15,8-100)$ & $60(14,6$ a 94,7$)$ & $100(47,8$ a 100$)$ \\
\hline
\end{tabular}


se recomendaba EFP e IFx tanto en suero como en orina. Sin embargo, los estudios en orina demostraron ser laboriosos, costosos y subjetivos ${ }^{5,6}$.

Creímos pertinente evaluar este panel (EFPs + CLLs) en nuestra institución, pensando en costos (inmunofijación y CLLs tienen un costo similar en nuestro medio) y en que la recolección de orina en 24 h es un examen difícil de realizar e interpretar, además de invasivo. Dado la diferencia en volumen, una muestra de suero tendrá menos errores que una muestra de orina de $24 \mathrm{~h}$. Adicionalmente, el ensayo de CLL ha mostrado tener mayor sensibilidad para la detección de proteínas monoclonales que la IFx en orina ${ }^{7}$.

Como era de esperar, el PS fue el de menor sensibilidad, principalmente, porque, en general, el único estudio solicitado fue EFPs. Este resultado nos pone en alerta, ya que puede plantearse una tasa de subdiagnóstico elevada, especialmente para amiloidosis AL y plasmocitomas (sensibilidad de $22 \%$ y $62,5 \%$ respectivamente). Esta bajísima sensibilidad en amiloidosis AL se ha visto antes y es ampliamente reconocido que el estudio de CLLs aumenta ostensiblemente su diagnóstico. En un estudio donde se analizaron 110 pacientes con amiloidosis, se observó que la medición de CLLs logró detectar el 91\% de los casos, contra 69\% de IFx y $83 \%$ de IFx orina ${ }^{8}$.

En cuanto a la sensibilidad del PI, destacamos que se realizó solo en los pacientes que contaban con IFx. Tal como se esperaba, PI mostró la mayor sensibilidad en detección de todas las GM, excepto amiloidosis, lo que se explica por la baja incorporación de IFx de orina en estos pacientes.

Por último, al analizar el panel "EFPs + CLLs", nuestros resultados muestran concordancia con lo observado por otros investigadores. Observamos una sensibilidad muy alta, aunque no enteramente comparable con el PI, probablemente por la diferencia en número de pacientes. Ya en 2006, Katzmann et al comprobaron, en su estudio sobre 428 pacientes, que las CLLs pueden reemplazar a la IFx orina al diagnóstico'. En 2009, Katzmann también sugirió un panel simplificado CLLs y EFPs cuando observó que no hubo disminución de sensibilidad en el diagnóstico de MM ni macroglobulinemia de Waldenström, en comparación con pacientes con todos los estudios en suero y orina ${ }^{7}$. Bakshi et $\mathrm{al}^{10}$ identificaron $39 \mathrm{GM}$ sobre 1.003 muestras utilizando EFPs, a lo que se adicionó la detección de 16 pacientes con incorporación de la evaluación de CLLs (tasa de incremento de detección de 41\%). Otro estudio similar mostró, sobre 312 pacientes analizados, un incremento de sensibilidad de 29\% al incorporar CLLs ${ }^{11}$.

\section{Conclusión}

En este escenario, la combinación "EFP + CLLs" es útil, ya que es el panel más simple con alta sensibilidad, sin requerir IFx ni muestras urinarias, laboriosas y subjetivas. Pensamos que la difusión de los resultados de este trabajo puede estimular a los médicos de la red pública de salud a utilizar un estudio diagnóstico sensible y temprano, para derivar oportunamente al especialista. Tenemos la certeza de que este estudio puede ayudar a incorporar al sistema público esta técnica, que ha demostrado mejorar el diagnóstico de nuestros pacientes.

\section{Referencias}

1. Bradwell AR, Carr-Smith HD, Mead GP, Tang LX, Showell PJ, Drayson MT, et al. Highly sensitive, automated immunoassay for immunoglobulin free light chains in serum and urine. Clin Chem 2001; 47: 673-80.

2. Beetham R, Wassell J, Wallage MJ, Whiteway AJ, James JA. Can serum free light chains replace urine electrophoresis in the detection of monoclonal gammopathies? Ann Clin Biochem 2007; 44: 516-22.

3. Dispenzieri A, Kyle R, Merlini G, Miguel JS, Ludwig H, Hajek R, et al. International Myeloma Working Group guidelines for serum-free light chain analysis in multiple myeloma and related disorders. Leukemia 2009; 23: 21524.

4. Rajkumar SV. International Myeloma Working Group updated criteria for the diagnosis of multiple myeloma. Lancet Oncology 2014; 15: e538-e48.

5. Hill PG, Forsyth JM, Rai B, Mayne S. Serum free light chains: an alternative test to Urine Bence Jones Proteins when screening for monoclonal gammopathies. Clin Chem 2006; 52: 1743-8.

6. Katzmann JA, Dispenzieri A, Kyle RA, Snyder MR, Plevak MF, Larson DR, et al. Elimination of the need for urine studies during diagnostic studies of monoclonal gammopathies by the combined use of serum immunofixation and serum free light chain assays. Blood 2006: $341 b$.

7. Katzmann JA, Kyle RA, Benson J, Larson DR, Snyder 
MR, Lust JA, et al. Screening Panels for Detection of Monoclonal Gammopathies. Clin Chem 2009; 55: 151722.

8. Katzmann JA, Abraham RS, Dispenzieri A, Lust JA, Kyle RA. Diagnostic performance of quantitative kappa and lambda free light chain assays in clinical practice. Clin Chem 2005; 51: 878-81.

9. Katzmann JA, Dispenzieri A, Kyle RA, Snyder MR, Plevak MF, Larson DR, et al. Elimination of the need for urine studies in the screening algorithm for monoclonal gammopathies by using serum immunofixation and free light chain assays. Mayo Clin Proc 2006; 81: 1575-8.

10. Bakshi NA, Gulbranson R, Garstka D, Bradwell AR, Keren DF. Serum free light chain (FLC) measurement can aid capillary zone electrophoresis in detecting subtle FLC-producing M proteins. Am J Clin Pathol 2005; 124: 214-8.

11. Abadie JM, Bankson DD. Assessment of serum free light chain assays for plasma cell disorder screening in a Veterans Affairs population. Ann Clin Lab Sci 2006; 36 : 157-62. 